\title{
Depolarization of Electronic Spin Qubits Confined in Semiconductor Quantum Dots
}

\author{
Dan Cogan, ${ }^{1}$ Oded Kenneth, ${ }^{1}$ Netanel H. Lindner, ${ }^{1}$ Giora Peniakov, ${ }^{1}$ Caspar Hopfmann, ${ }^{1}$ \\ Dan Dalacu, ${ }^{2}$ Philip J. Poole, ${ }^{2}$ Pawel Hawrylak, ${ }^{3}$ and David Gershoni ${ }^{1, *}$ \\ ${ }^{1}$ The Physics Department and the Solid State Institute, Technion-Israel Institute of Technology, \\ 3200003 Haifa, Israel \\ ${ }^{2}$ National Research Council of Canada, Ottawa, Ontario, Canada K1A OR6 \\ ${ }^{3}$ Physics Department, University of Ottawa, Ontario, Canada Ontario Canada K1N 6N5
}

(Received 5 August 2018; revised manuscript received 26 October 2018; published 18 December 2018)

\begin{abstract}
Quantum dots are arguably the best interface between matter spin qubits and flying photonic qubits. Using quantum dot devices to produce joint spin-photonic states requires the electronic spin qubits to be stored for extended times. Therefore, the study of the coherence of spins of various quantum dot confined charge carriers is important both scientifically and technologically. In this study we report on spinrelaxation measurements performed on five different forms of electronic spin qubits confined in the very same quantum dot. In particular, we use all optical techniques to measure the spin relaxation of the confined heavy hole and that of the dark exciton-a long-lived electron-heavy-hole pair with parallel spins. Our measured results for the spin relaxation of the electron, the heavy hole, the dark exciton, the negative and the positive trions, in the absence of externally applied magnetic field, are in agreement with a central spin theory which attributes the dephasing of the carriers' spin to their hyperfine interactions with the nuclear spins of the atoms forming the quantum dots. We demonstrate that the heavy hole dephases much slower than the electron. We also show, both experimentally and theoretically, that the dark exciton dephases slower than the heavy hole, due to the electron-hole exchange interaction, which partially protects its spin state from dephasing.
\end{abstract}

DOI: 10.1103/PhysRevX.8.041050

Subject Areas: Condensed Matter Physics,

Quantum Information

\section{INTRODUCTION}

The electronic spin in semiconductor nanostructures can often be described as an isolated physical two-level system. As such it has long been considered an excellent qubit with great potential to be used in future quantum informationprocessing-based technologies [1,2]. Moreover, semiconductor nanostructures, which confine single electrons, are easily integrated into electronic and optical devices and circuits, which dovetail with the contemporary semiconductor-based electro-optic technology. Therefore, much effort has been devoted recently to demonstrate that various forms of the electronic spin in semiconductor nanostructures and in particular in quantum dots (QDs) can be initiated and controlled with relatively high fidelities, using optics and electronics means [3-6]. An important advantage of semiconductor electronic spin qubits, which are

\footnotetext{
*dg@physics.technion.ac.il
}

Published by the American Physical Society under the terms of the Creative Commons Attribution 4.0 International license. Further distribution of this work must maintain attribution to the author(s) and the published article's title, journal citation, and DOI. anchored to the device, is their strong interaction with photons, which can be used as flying qubits to communicate quantum information to remote locations [7-10]. These advantages have been recently used, for instance, to demonstrate that a QD confined electronic spin, can be used as an entangler for on-demand production of a long string of entangled photons in a cluster state [11].

The main decoherence mechanism of the confined electronic spin (central spin) in semiconductor QDs is its interaction with the spins of the nuclei in its vicinity [12-17]. Therefore, it is essential, both scientifically and technologically, to study and to characterize these dephasing processes.

In this work, we comprehensively study, both experimentally and theoretically, the dephasing dynamics of QD confined electronic spins in five different forms: (a) conduction-band electron, (b) valence-band heavy hole, (c) negative trion, (d) positive trion, and (e) dark exciton (DE). All in the same single QD.

Semiconductor QDs are formed by about $10^{5}$ molecules of one semiconductor compound embedded in another semiconductor compound of higher band-gap energy. These formations give rise to nanometer-scale threedimensional (3D) potential traps, which confine single 
electronic charge carriers (electrons in the conduction bands and holes in the valence bands) and isolate them from their environment. The energy spectrum of these confined carriers is therefore discrete, giving rise to welldefined and spectrally sharp optical transitions between these discrete levels $[18,19]$.

In Fig. 1 we display the electronic spin-wave functions and Bloch-sphere representations of all the electronic spin qubits used in this work. The confined conduction electron levels have a vanishing atomic orbital momentum and thus their total spin projection on the QD growth direction is $\pm 1 / 2$. Therefore, they form physical two-level systems or qubits [20]. The spin state of the qubit is represented on the Bloch sphere, where the spin-up and the spin-down states are located at the north and south poles of the sphere, respectively, and any superposition of these two states is represented by a point on the sphere's surface. The confined valence-band electron states have a total atomic orbital momentum of 1 . The spin-orbit interaction, together with the quantum confinement along the growth direction and the biaxial lattice mismatch compressive strain, inherent to our strain-induced QDs, results in a large energy splitting between the upper most valence states [21]. The highest valence electron states in which the orbital spin and electronic spin are parallel, are few tens meV higher than the states in which the orbital and electronic spins are antiparallel. At low temperature, the valence-band states are fully occupied. Confined positive charge carriers in the QD are therefore formed due to the absence of valence-band electrons. Thus, the lowest energy hole states have angular momentum projection of $\pm 3 / 2$ on the growth direction (heavy holes). A heavy hole, is yet another form of a QD confined electronic spin qubit $[22,23]$ as shown in Fig. 1. Another form of a confined electronic spin qubit is the electron-heavy-hole pair, or the exciton [24,25]. Excitons in which the heavy-hole spin and the electron spin are antiparallel have a total spin projection of \pm 1 , they are optically active and therefore called bright excitons (BEs). The qubit that they form [24-26] recombines within a short radiative lifetime (about $1 \mathrm{~ns}$ ), which limits their use as a matter spin qubit. In contrast, excitons in which the electron and heavy-hole spins are parallel, are optically inactive since the electromagnetic radiation barely interacts with the electronic spin. These excitons are called dark excitons. They have a total spin projection of \pm 2 on the QD growth axis and live orders of magnitude longer than the BE [27]. Consequently, they can be used for implementing sophisticated quantum information protocols [11,28,29].

In the following, we denote these three long-lived forms of spin qubits (electron, heavy hole, and DE) - groundlevel qubits. The ground-level qubits are stable, and once generated in the QD they live in it for a very long time. The ground-level qubits can be optically excited to their respective excited-level qubits by absorbing a single photon, which adds an electron-hole pair to the QD.
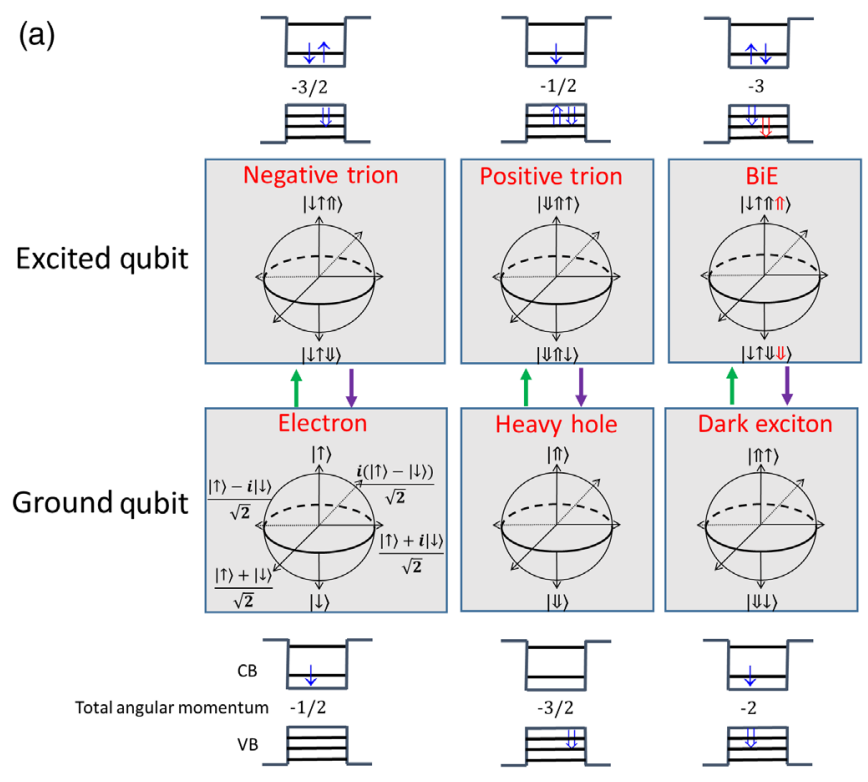

(b)
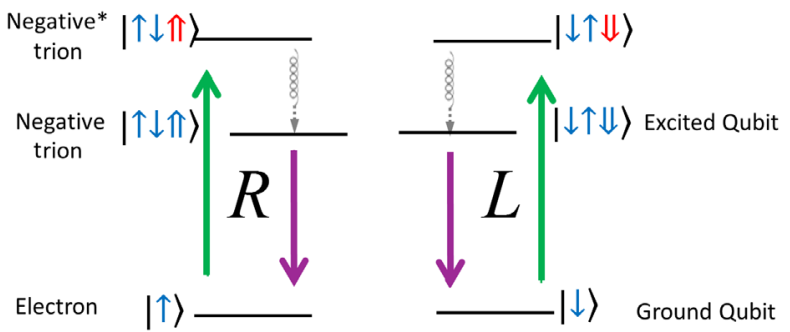

FIG. 1. (a) Spin wave functions and Bloch-sphere representations of the six matter spin qubits used in this work. The six qubits, represented by their Bloch spheres, are divided into three pairs of ground and excited-level qubits. The spin wave functions of the ground-level (excited-level) qubits are schematically described below (above) the respective Bloch spheres, where $\uparrow(\Downarrow)$ represents spin-up electron (down heavy hole), and the blue (red) color represents a carrier in its ground (excited) energy level. Green upward arrows represent laser pulses which convert the ground-level qubit to its respective excited-level qubit. Magenta downward arrows represent single photons emitted from the excited qubits thereby returning to the ground-level qubit. (b) The optical transitions and polarization selection rules for the electron-trion system, which form a ground-level-excited-level qubit pair. Note that in this example (as in all other cases) an optical $\Pi$ system is described, but the exciting laser pulse is tuned to an excited trion level, in order to facilitate polarization tomography of the emitted photon (magenta downward arrows) by spectrally separating the emission from the exciting laser pulse (green upward arrows). The fast (about 70 ps [29]) phononassisted relaxation of the excited trion to the ground trion level is represented by gray curly downward arrows. The right-hand (lefthand) circular polarization of the photons which connect the $1 / 2$ $(-1 / 2)$ spin state of the ground-level qubit with the $+3 / 2(-3 / 2)$ spin state of the excited qubit are marked by $R(L)$.

Moreover, by using a resonantly tuned optical $\pi$ pulse, this excitation can be done deterministically. The resonant excitation converts the ground-level qubits to their 
excited-level qubits, as schematically described in Fig. 1. In Fig. 1, green upward arrows represent the optical laser excitations, which convert the electron spin qubit to the negative trion qubit, the heavy-hole qubit to the positive trion qubit, and the DE to the spin-blockaded biexciton qubit. As can be seen in Fig. 1, the negative and positive trion qubits are formed by three carriers. The negative trion is formed by two ground-level conduction-band electrons in a singlet state and a single ground-level heavy hole, while the positive trion is formed by two ground-level heavy holes and a single ground-level electron. In both cases, the spin state of the trion qubits is determined by the minority carrier, $\pm 3 / 2$ for the negative trion, and $\pm 1 / 2$ for the positive trion.

Unlike the trions, which are formed by three carriers, the biexciton is formed by four carriers. Two ground-level electrons in a singlet spin state, and two heavy holes with parallel spins in the ground and first excited valence-band levels. Consequently, the biexciton-qubit spin states are \pm 3 , and it is determined by the two parallel heavy holes' spin directions.

Once formed, the excited spin qubits, which are optically active, decay radiatively within the radiative lifetime of a ground-level electron-hole pair (about $1 \mathrm{~ns}$ ), by emitting a single photon and the system returns to the ground-level qubit. The photon emissions are schematically described by the downward magenta arrows in Fig. 1.

If the upper qubit is properly initialized in a coherent superposition of its two spin states, the polarization of the emitted photon ("flying photonic qubit") is expected to be entangled with the spin state of the ground-level spin qubit, which remains in the QD [8-11].

At low temperatures and in the absence of external magnetic field, the main decoherence mechanism of these electronic spin qubits is the hyperfine interaction between the electronic (central) spin and the spin of the nuclei of the approximately $10^{5}$ atoms which form the QDs [14-16]. The two types of charge carriers in semiconductors, the negative conduction-band electrons, and the positive, valence-band holes interact differently with the nuclei, since their orbital momentum around the nucleus is different. The conduction electrons have zero atomic orbital momentum, while valence-band holes have unit atomic orbital momentum. Consequently, the conduction electron's wave function strongly overlaps with the nucleus and interacts with the nuclear spin via the Fermi contact interaction. In contrast, the valence hole's wave function vanishes at the nucleus site and therefore its spin interacts with the nuclear spin via the weaker dipole-dipole hyperfine interaction [17]. In addition, while the conductionelectron interaction with the nuclei, which we denote by $\gamma_{e}$, is isotropic, the interaction of the valence heavy hole for which the orbital angular momentum and the spin are aligned parallel to the growth direction, is anisotropic. We denote by $\gamma_{h_{z}}$ the interaction of the valence heavy-hole spin with the nuclei spin bath along the QD growth axis $(\hat{z})$ and by $\gamma_{h_{p}}$ the interaction with nuclear spins in the plane perpendicular to $\hat{z}$.

The dynamics of the electronic central spin can be divided into two different time domains as schematically described in Figs. 2(a)-2(c) for the electron, heavy hole, and DE spins, respectively [15].

During the first stage, the central spin precesses around a mean effective magnetic field generated by the frozen fluctuations of the nuclear spins in its vicinity. The electron interacts with the nuclear field via the isotropic Fermi contact hyperfine interaction marked by $\gamma_{e}$, while the heavy hole interacts via the anisotropic dipole-dipole hyperfine interaction marked by $\gamma_{h_{z}}$ and $\gamma_{h_{p}}$. As the DE is formed by an electron-hole pair with parallel spins, each of these carriers interacts with the nuclear magnetic field, while at the same time they also interact with each other, via the electron-hole exchange interactions. The most important term in this interaction is the isotropic term $\Delta_{0}[21,30]$, separating the DE and BE (an antiparallel electron-hole pair) energy levels. Being much stronger than the hyperfine interactions it prevents the separate spin flip of either one of the two individual spins and consequently protects the $\mathrm{DE}$ spin from dephasing. It turns out, as we show in Appendix B, below, that the DE nuclear field-induced dephasing is caused mainly due to small DE-BE mixing terms (of order $10^{-3}$ ).

During the second stage, at longer times, the fluctuations in the nuclear magnetic field can no longer be considered "frozen" and they slowly evolve in time. This evolution is described as local precession of the effective magnetic field around local directions denoted by $\hat{n}$. A relatively simple model describes this motion as generated by the quadrupole interaction (denoted by $\gamma_{Q}$ ) of the nuclear spins with the strain-induced electric field gradients in the QD [31-34]. We adopt this description, since it permits analytic solution to the problem, thereby simplifying the comparison with the measured data, while keeping the generality of our approach. Finally, at yet longer times, which is beyond the scope of this work, the nuclei also interact with each other via the dipole-dipole nuclear interaction [35]. During the second stage the central spin continues to interact with the slowly varying effective nuclear magnetic field in the same manner as it does during the first stage. Therefore, the central spin dynamics can be described as a sort of "convolution" between the relatively fast dynamics of the spin around the average nuclear magnetic field, with the dynamics of the slowly varying nuclear field.

The details of the model involved in these calculations, which follow Refs. [15,31,33], describing the evolution of the electron, and the generalization of the model to include the heavy-hole evolution, are described in Appendix A. The model, which describes the dynamics of the DE, is developed in Appendix B. 


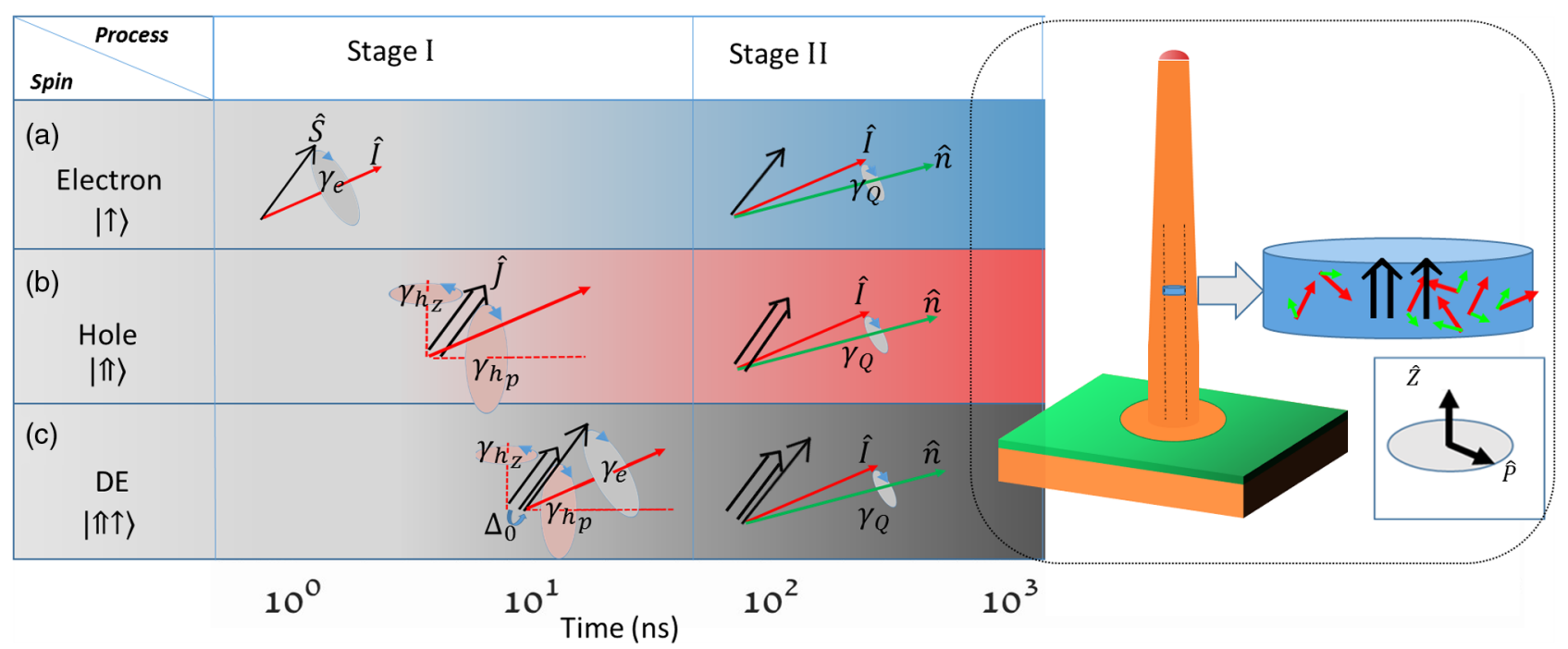

FIG. 2. Schematic description of the spin dephasing processes of the QD confined electron (a), heavy hole (b), and dark exciton (c). Each process is divided into two temporal stages: During the first stage the initiated central spin precesses around the effective magnetic field which results from the frozen fluctuation of the nuclear spins of about $10^{5}$ atoms comprising the QD. The electron spin $(\hat{S})$ interacts with the nuclear spin $(\hat{I})$ via the isotropic Fermi contact interaction described by $\gamma_{e}$. The heavy-hole spin $(\hat{J})$ interacts with the nuclear spin via the anisotropic dipole-dipole hyperfine interaction denoted by $\gamma_{h_{z}}$ and $\gamma_{h_{p}}$, where $\hat{z}$ is the QD growth direction and $p$ denotes direction in a plane perpendicular to $\hat{z}$. The dark exciton contains an electron and a heavy hole. Both spins interact with the nuclear spin, and in addition, the electron and hole interact with each other mainly via the isotropic exchange interaction denoted by $\Delta_{0}$ [30]. During the second stage the nuclear spins react to strain-induced electric field gradients (EFG) in the QD [31]. This interaction has a quadrupole nature, and we denote it by $\gamma_{Q}$. The motion of each nuclear spin is described by an effective local magnetic field in a direction marked by $\hat{n}$ which the nuclear spin slowly precesses around. During the second stage, we use the adiabatic approximation, by which the central spin just follows the slowly varying effective nuclear magnetic field. The various interaction magnitudes are summarized and referenced in Table I. Inset: schematic description of the InAsP QD (blue) embedded in the InP photonic nanowire (orange). The central spin is represented by the black arrows, the nuclear spin bath are represented by the red arrows, and the EFGs are schematically represented by green arrows in the magnified description of the QD.

A great deal of effort was devoted to study the coherence properties of the central electronic spin for both, conduction-band electrons [36-39], and valence-band heavy holes [22,40-45], confined in QDs. The temporal evolution of a single electron spin at vanishing external magnetic field was experimentally measured recently by Bechtold and co-workers [33]. To the best of our knowledge, similar measurements for the heavy hole as a central spin have not been reported so far. Here, we present comprehensive measurements of the spin depolarization dynamics for both the electron and the heavy hole as well as for their correlated pair - the DE. All these forms of central electronic spin are confined to the same QD. In addition, we show, by measuring the temporal evolution of the positive and negative trions' spins, that the presence of two additional paired charge carriers does not affect the central spin depolarization. Our measurements are preformed optically without applying any external magnetic field. In addition, we carry out the experiments in a way which prevents the generation of a steady-state nuclear Overhauser field or influencing fluctuations in its distribution [46-50]. The experimental methods and measurements are described below and the measured results are compared with the central spin models discussed in the Appendices.

\section{THE DEVICE AND EXPERIMENTAL METHODS}

The InP nanowire containing a single InAsP quantum dot [51-53] is grown using chemical beam epitaxy with trimethylindium and precracked $\mathrm{PH}_{3}$ and $\mathrm{AsH}_{3}$ sources. The nanowires are grown on a $\mathrm{SiO}_{2}$-patterned (111)B InP substrate consisting of circular holes opened up in the oxide mask using electron-beam lithography and a hydrofluoric-acid wet etch. Gold is deposited in these holes using a self-aligned lift-off process, which allows the nanowires to be positioned at known locations on the substrate. The thickness of the deposited gold is chosen to give 20-nm to 40-nm diameter particles, depending on the size of the hole opening. The nanowires are grown at $420^{\circ} \mathrm{C}$ with a trimethylindium flux equivalent to that used for a planar InP growth rate of $0.1 \mu \mathrm{m} / \mathrm{h}$ on (001) InP substrates at a temperature of $500{ }^{\circ} \mathrm{C}$. The growth is a twostep process: (i) growth of a nanowire core containing the quantum dot, nominally $200 \mathrm{~nm}$ from the nanowire base, and (ii) cladding of the core to realize nanowire diameters (around $200 \mathrm{~nm}$ ) for efficient light extraction. The quantum dot diameters are determined by the size of the nanowire core. The particular QD reported on here has a diameter of about $30 \mathrm{~nm}$. 
The sample is placed inside a sealed metal tube cooled by a closed-cycle helium refrigerator maintaining a temperature of $4 \mathrm{~K}$. A $\times 60$ microscope objective with numerical aperture of 0.85 is placed above the sample and used to focus the laser beams on the sample surface and to collect the emitted PL from it. Pulsed laser excitations are used. The picosecond pulses are generated by two synchronously pumped dye lasers at a repetition rate of $76 \mathrm{MHz}$. The temporal width of the pulses is $12 \mathrm{ps}$ and their spectral width about $100 \mu \mathrm{eV}$. Light from a continuous wave (CW) laser, modulated by an acousto-optic modulator, synchronized with the dye lasers, is used to produce pulses of up to 30-ns duration. These pulses are used to set the average QD charge state [54]. A second CW laser, modulated by an electro-optic modulator, is used to produce depletion pulses of 30-ns duration [55]. The timing between the two synchronized picosecond pulses is controlled using two cavity dumpers which effectively reduce the repetition rate down to $0.5 \mathrm{MHz}$. In addition, a computer-controlled motorized delay line is used to finely tune the temporal delay between the pulses. The polarizations of the excitation pulses are independently adjusted using polarized beam splitters (PBS) and two pairs of computer-controlled liquid crystal variable retarders (LCVRs) [11]. The collected PL is equally divided into two beams by a nonpolarizing beam splitter. Two pairs of LCVRs and a PBS are then used to analyze the polarizations of each beam. In this way the emitted PL is divided into four beams, allowing the selection of two independent polarization projections and their complementary polarizations. The PL from each beam is spectrally analyzed by either a 1 or 0.5 meter monochromator and detected by a silicon avalanche photodetector coupled to a PicoQuant HydraHarp 400 time-correlated photon counting and time tagging system, synchronized with the pulsed lasers. In this way the arrival times of up to four emitted photons have been recorded with respect to the synchronized laser pulses.

We use the optical transitions between the ground-level qubits and the excited-level qubits to initialize the spin state of both qubits, and then for probing the spin state of the qubits at a later time. We facilitate the optical transition selection rules of the $\Pi$ systems described in Fig. 1(b) in order to do that.

For initializing the excited qubit, one simply applies an $R$ or $L$ polarized $\pi$ pulse. For probing the excited qubit spin projection, one simply measures the degree of circular polarization of the emitted photons $\hat{S}_{z}=$ $\left(I_{R}-I_{L}\right) /\left(I_{R}+I_{L}\right)$ where $I_{R(L)}$ is the measured emission intensity projected on right-hand (left-hand) circular polarization.

The initialization of the ground-level qubit is provided by detecting $R$ or $L$ polarized single photon, which heralds the spin state of the qubit at the photon emission time. Probing the ground-level qubit spin state is done by first converting the state into the state of the excited-level qubit, using an horizontally linearly polarized $[H=(R+L) / \sqrt{2}]$ $\pi$ pulse, and then measuring the time-resolved degree of circular polarization of the emitted photons. For example, in Fig. 1(b) if the electron spin state before the pulse is described by $\hat{\rho}_{\text {electron }}=p\left|\Psi_{\text {electron }}\right\rangle\left\langle\Psi_{\text {electron }}\right|+(1-p) \frac{1}{2} \mathbb{I}$, where $\mathbb{I}$ is the identity matrix and $p$ is the probability of $\hat{\rho}_{\text {electron }}$ being in a pure state $\left|\Psi_{\text {electron }}\right\rangle=\alpha|\uparrow\rangle+\beta|\downarrow\rangle$, then after the pulse the photogenerated trion spin state is given by $\hat{\rho}_{\text {trion }}=p\left|\Psi_{\text {trion }}\right\rangle\left\langle\Psi_{\text {trion }}\right|+(1-p) \frac{1}{2} \mathbb{I}$, with $\left|\Psi_{\text {trion }}\right\rangle=\alpha|\uparrow \downarrow \uparrow\rangle+\beta|\downarrow \uparrow \downarrow\rangle$, with the same $\alpha, \beta$, and $p$. Here, we assume, of course, that the fidelity of the optical excitation by the $H$ polarized $\pi$ pulse is unity and that the experimental deviation from truly $H$ polarization is negligible. The spin projection of excited qubit on the $\hat{z}$ direction is then deduced by measuring the degree of circular polarization of the emitted photons.

We conduct five different experiments in order to comprehensively study the central spin dynamics for various confined spin qubits in the QD. In the first two measurements, schematically described in Fig. 3(a), we measure the depolarization of the negative or positive trions. We first pump the QD to either a negative or a positive charge state by using above band-gap CW1 pulse of about 20-ns duration [54]. Then, either an excited negative or positive trion is photogenerated by using a short circularly polarized quasiresonant approximately 12-ps-long laser pulse. The polarization of the excitation pulse determines the spin polarization of the minority carrier in the initialized trion [hole (electron) in the negative (positive) trion]. After a fast (about 70 ps [29]) spin preserving phonon assisted relaxation of the excited trion, a ground-level trion is formed. When the trion decays radiatively, the polarization of the emitted photon reflects the spin of the minority carrier at the particular time in which the photon is emitted. Thereby, by using time-resolved circular polarization-sensitive PL measurements we probe the spin relaxation dynamics of the minority carrier in the trion. This technique provides a simple way of measuring the dynamics of the spin of the confined electron (hole) in the presence of a spin singlet pair of two holes (electrons). Unfortunately, this simple method is limited by the relatively short radiative lifetime of the trion. Only the evolution during the first time domain can be measured this way. In order to avoid generating a steady-state Overhauser field in the QD due to the repeated circularly polarized quasiresonant excitation pulse, a second pulse with opposite circular polarization is used to reexcite the trion a few nanoseconds after the first pulse, during the same excitation period. The time-resolved degree of circular polarization is deduced using the resulted PL from both pulses. As mentioned above, the circularly polarized resonant excitation pulses are preceded by a 20 -ns-long, nonresonant and linearly polarized excitation pulse (CW1). The CW1 pulses determine the average charge state of the QD, but in 

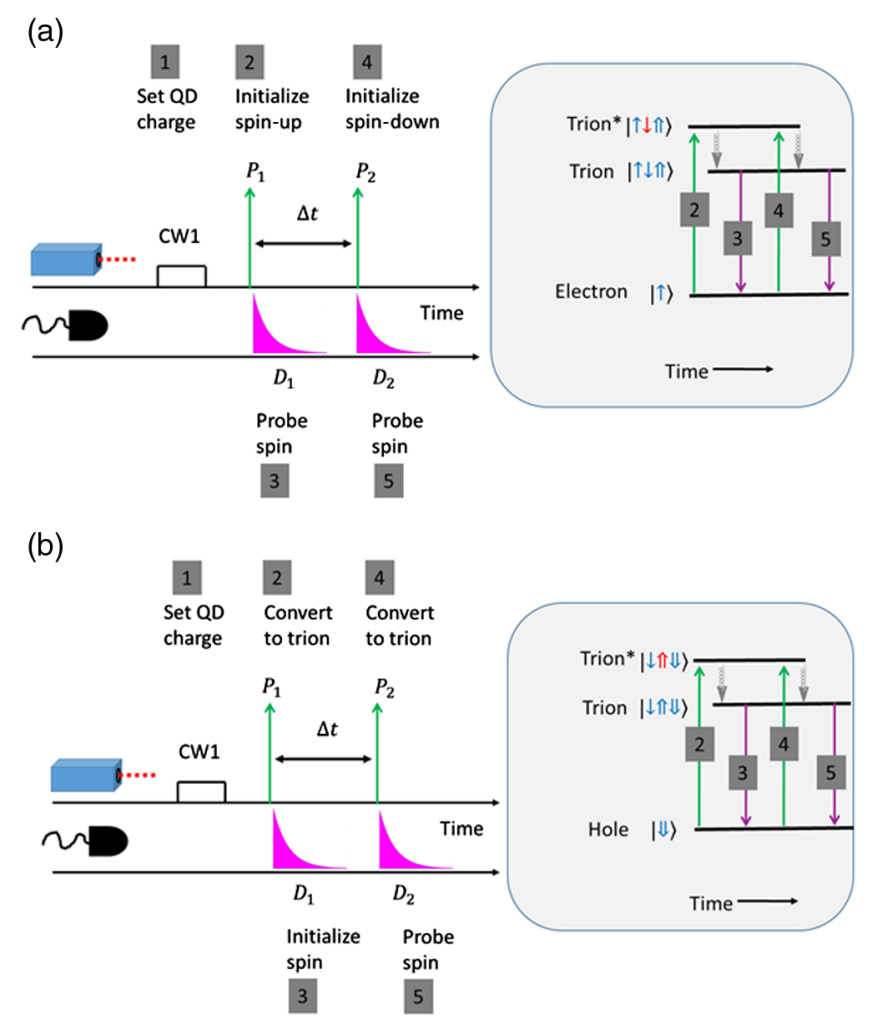

(c)

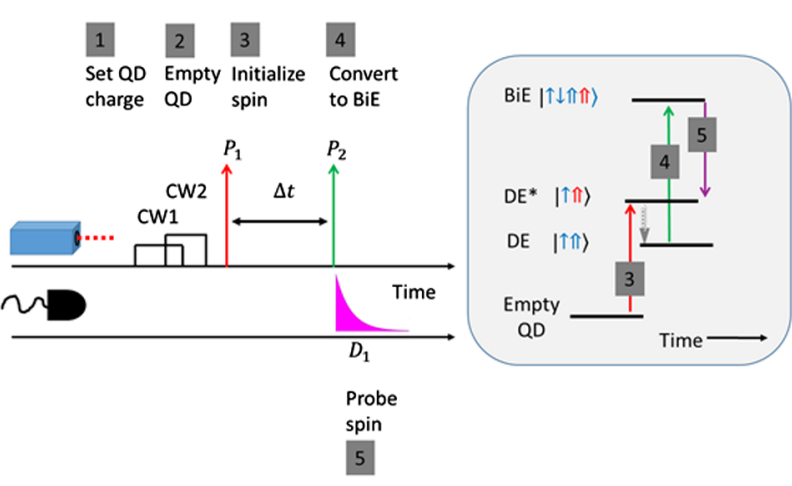

FIG. 3. Schematic description of the experiments for measuring the spin dynamics of (a) positive and negative trions, (b) single electron and single heavy hole. (c) Dark exciton. The optical transitions in each experiment are described by the energy-level diagram to the right. The carrier's spins are marked in the figure using the notations of Fig. 1, where blue (red) color represents ground (excited) single carrier states. CW1 and CW2 represent the 20-ns gated CW laser pulses where $P_{1}$ and $P_{2}$ represent the 12 -ps pulses produced by the synchronously pumped and cavitydumped dye lasers. $\Delta t$ is the time delay between the two pulses in each repetition period, controlled by two cavity dumpers and a delay line. $D_{1}$ and $D_{2}$ represent the emission and time-resolved detection of the two single photons emitted as a result of the $P_{1}$ and $P_{2}$ excitations.

addition further prevent the buildup of a nuclear magnetic field in the QD.

The measurement of the spin dynamics of either the single electron or heavy hole is carried out using the same

experimental system but in a somewhat different manner, as schematically described in Fig. 3(b). In the inset to this figure we describe the energy levels of the heavy-hole system. Here, after the optical charging, a trion is generated by quasiresonant excitation using a horizontal $(H)$ polarized pulse. Either the electron or the hole spin is initialized by detecting the circular polarization of the emitted single photon. In order to probe the temporal dependence of the spin state of the carrier, a second, horizontal polarized delayed 12-ps pulse is used to reexcite the carrier to its respective trion and the resulting circular polarization of the emitted photon is used to measure the spin polarization of the carrier at the reexcitation time. This measurement is not limited by the radiative lifetime of the trion, however, it requires two photon intensity correlation measurements in a relatively slow repetition rate (about $500 \mathrm{kHz}$ ). We achieve this low repetition rate by using the cavity dumpers. The feasible maximal delay time (about $1 \mu \mathrm{s}$ ) between the pulses is defined by the rejection ratio (of about $2 \times 10^{-3}$ ) of neighboring pulses of the cavity dumpers. Note that in these experiments the generation of an Overhauser field is avoided because the initialization of the central spin is not done deterministically by using circularly polarized excitation, but rather probabilistically by postselecting the detected circular polarization of the emitted first photon.

The spin dynamics of the DE is probed as schematically described in Fig. 3(c). Here, we use above-band-gap optical pumping of about $20 \mathrm{~ns}$ to neutralize the QD and then another quasiresonant pumping of about $20 \mathrm{~ns}$ to deplete the QD from the DE [55]. After depleting the QD, a quasiresonant circularly polarized 12-ps pulse initializes the DE in spin-up excited state [56]. Following this initialization, the DE relaxes to its ground state within approximately $70 \mathrm{ps}$ by spin-preserving emission of a phonon. In order to probe the DE state, a delayed, linearly polarized resonant 12-ps pulse converts the DE qubit into the biexciton qubit. Note that the horizontal polarization of the laser preserves the phase of the qubit. The detection of a circularly polarized photon, which results from the radiative recombination (about 1-ns lifetime) of the biexciton is then used to probe the spin state of the DE in the QD, at the converting pulse time. Repetition rates as low as about $500 \mathrm{kHz}$, allow temporal delays of over $1 \mu$ s between initialization and probing of the spin. In this experimental method an Overhauser field is not generated in the sample since the gated CW pulses used to optically pump and deplete the QD are linearly polarized.

\section{RESULTS AND DISCUSSION}

In Fig. 4 we present the measured degree of the average central spin polarization $\left\langle S_{z}(t)\right\rangle$ as a function of time after its initialization, for the five spin qubits: the conductionband electron, the valence-band heavy hole, the positive and negative trions, and the dark exciton. The error bars represent one standard deviation of the experimental 


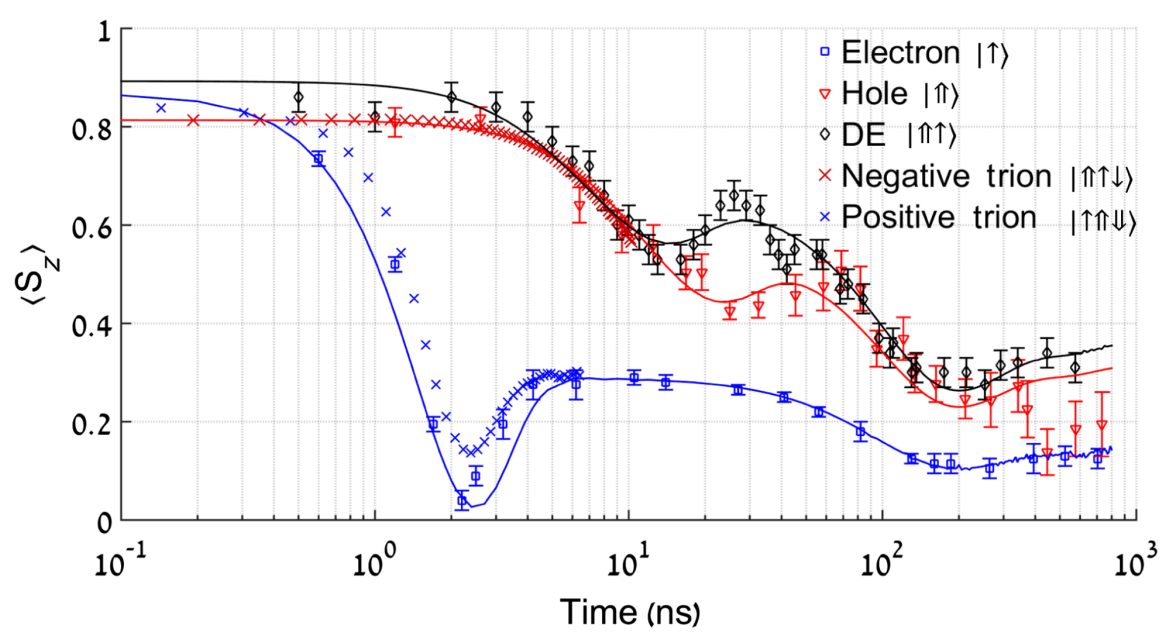

FIG. 4. Measured central spin polarization $\left\langle S_{z}\right\rangle$ as a function of time after its initialization, for the QD confined electron (blue square symbols), heavy hole (red triangle symbols), dark exciton (black diamond symbols), negative trion (red times symbols), and positive trion (blue times symbols). Error bars represent one standard deviation of the experimental uncertainty. Solid color-matched lines represent the fitted theoretical model (see Appendices), for each case. The spin wave functions are schematically described in the legend, where the notations of Fig. 1 are used.

uncertainty. At time zero the central spin is initialized to the spin-up state. Then, the projection of the spin on the $\hat{z}$ direction (the QD growth axis) is displayed as a function of time.

The conduction-band electron spin state (blue rectangles) depolarizes from its initial state within about $2 \mathrm{~ns}$. The spin polarization then revives to about a third of the initial polarization. From this level the polarization continues to decay at a much slower rate, reaching a second minimum at about 200 ns. Afterwards, the spin polarization revives again to about $10 \%$ of the initial polarization. This behavior is similar to that reported in Ref. [33], as predicted by Ref. [15]. Roughly speaking, the first fast dephasing step is a measure for the strong Fermi-contact hyperfine interaction of the electron with the nuclear spin bath, while the second step measures the strength of the quadrupole interaction of the nuclear spin bath with the strain-induced electric field gradients in the QD.

After initialization, the heavy hole (red triangles) spin depolarizes in about an order of magnitude slower than the electron spin. This is due to the much weaker dipole-dipole hyperfine interaction. The hole spin polarization decreases at approximately $20 \mathrm{~ns}$ to about one-half of its initial polarization. Afterwards, it mildly revives followed by a slow decay due to the quadrupole interaction of the nuclear bath.

The positive trion spin polarization (blue times symbols), behaves similarly to that of the electron, while the negatively charged trion spin polarization (red times symbols) follows that of the heavy hole. This is not surprising, since the trion polarization reflects the polarization of the unpaired minority carrier, in the presence of the two paired majority carriers. As explained above, the trion spin measurements are limited by their radiative lifetime of about $1 \mathrm{~ns}$.
The dark exciton (black diamonds) decoheres slowly, in a similar rate to the heavy hole. However, like the electron, after the initial decay, it strongly revives to about two-thirds of its initial polarization. This is due to the strong exchange interaction between the electron and hole that protects both carriers from flipping their individual spins. Later, after about $200 \mathrm{~ns}$, the dark-exciton polarization continues to decay due to the quadrupole interaction.

We fit the measured temporal behavior of the electron, heavy hole, and dark exciton using one conceptually simple central spin model. For the fitting, only five free parameters are used: (1) The hyperfine Fermi-contact interaction $\gamma_{e}$, (2) the heavy-hole out-of-plain hyperfine dipole-dipole interaction $\gamma_{h_{z}}$, (3) the heavy-hole in-plain hyperfine dipole-dipole interaction $\gamma_{h_{p}}$, (4) the DE in-plane interaction $\gamma_{\mathrm{DE}_{p}}$, and (5) the quadrupole interaction $\gamma_{Q}$. These parameters are accurately defined in the Appendices, where the models are discussed for the electron and the heavy hole (Appendix A), and for the DE (Appendix B).

The best-fitted parameters are given in Table I, where they are also compared with the available literature. Our analysis provides an estimation of the number of atoms in the QD: $N_{L}=3 \times 10^{5}$. With this estimation our fitted hyperfine Fermi contact $\gamma_{e}$ is comparable to that of Ref. [57].

Characteristic spin depolarization times during the first and second temporal stages can be obtained from our fitting procedure quite straightforwardly. Since the central spins in this work are initialized in the $z$ direction, depolarization is caused by the in-plane interaction parameters. Thus, the temporal location of the first minimum is a rough measure of the in-plane interaction parameter: $T_{\min }=\hbar / \gamma_{p} \sim 2,20$, and $14 \mathrm{~ns}$ for the electron, heavy hole, and DE, respectively. 
TABLE I. The various interaction energies as obtained by the best model fitting to the measured temporal depolarization of five different electronic spin qubits confined in the same QD. The theoretical model and the fitted parameters are described in the text and the Appendices.

\begin{tabular}{llc}
\hline \hline Interaction & This work $(\mu \mathrm{eV})$ & Literature $(\mu \mathrm{eV})$ \\
\hline$\gamma_{e}$ & $0.34 \pm 0.03$ & $0.33[33]$ \\
$\gamma_{h_{z}}$ & $0.11 \pm 0.03$ & $0.081[41]$ \\
$\gamma_{h_{p}}$ & $0.031 \pm 0.006$ & $0.047[41]$ \\
$\gamma_{\mathrm{DE}_{p}}$ & $0.047 \pm 0.006$ & $\ldots$ \\
$\gamma_{Q}$ & $0.0031 \pm 0.001$ & $0.00087[33]$ \\
\hline \hline
\end{tabular}

Thus, $\gamma_{e}, \gamma_{h_{p}}$, and $\gamma_{\mathrm{DE}_{p}}$ are given by $0.34,0.031$, and $0.047 \mu \mathrm{eV}$, respectively.

The central spin interaction with the nuclear field along the $z$ direction, acts as a restraining force, which actually prolongs the spin coherence. Therefore, roughly speaking, the ratio between these interactions $\left(R_{\gamma}=\gamma_{Z} / \gamma_{P}\right)$ determines the depth of the first polarization minimum and the maximum value of the polarization after its revival. We thus obtain $R_{\gamma}=1,3.5$, and 5, for the electron, hole, and DE, respectively. Note that for the electron the ratio is by definition 1 , and therefore the polarization degree revives to $1 / 3$ of its initial value, while for the hole and $\mathrm{DE}$ it revives to higher values. During the second temporal stage, the polarization of all three central spins decays more or less at the same rate, determined by the quadrupole interaction $\gamma_{Q}$. Therefore, the temporal location of the second minimum is about the same in all cases given by $T_{\min _{Q}}=\hbar / \gamma_{Q} \sim$ $200 \mathrm{~ns}$ or $\gamma_{Q} \sim 0.003 \mu \mathrm{eV}$. The fact that three different experimental measurements yielded the same value for $\gamma_{Q}$ further indicates that the optical excitations do not create a net nuclear magnetic field or affect the statistical distribution of the field fluctuations.

The quadrupolar coupling that we deduce is larger by about a factor of 3 than that reported by Bechtold et al. [33]. We attribute this difference to the difference in the QD material system studied in both cases, InAsP here vs InGaAs, in Ref.[33], as further explained in Appendix A below.

A common practice for quantifying the depolarization value of a spin qubit is to define the depolarization time as the time it takes for the polarization to reduce to $1 / e$ of its initial state. We adopt this practice, though the measured depolarizations are clearly nonexponential. The measured depolarization times thus obtained are 1.5, 130, and $145 \mathrm{~ns}$ for the electron, heavy hole, and DE, respectively.

\section{SUMMARY}

We investigate both experimentally and theoretically the depolarization dynamics of five different electronic spin configurations confined in the same semiconductor quantum dot. Our measurements are carried out all optically and in the absence of externally applied magnetic field. We show that the measured temporal spin depolarization is well described by a central spin model which attributes the depolarization to the hyperfine interaction between the electronic spin and the nuclear spin bath of the QD atoms.

We divide the depolarization into two temporal stages. During the initial stage the central spin precesses around the effective magnetic fields of the frozen fluctuations of the $10^{5}$ nuclear spins in the QD. During the second stage the central spin precession follows adiabatically the nuclear spin bath dynamics which ceases to be frozen and effectively precesses around strain-induced electric field gradients in the QD.

These two processes result in a relatively fast initial depolarization of the central spin reaching a first minimum. The depolarization minimum is then followed by a temporal revival of the polarization degree and finally by a second depolarization reaching a minimum at a much later time which is more or less equal for all the electronic central spin cases.

Our model assumes that while the hyperfine interaction between the central spin and the nuclear spins is isotropic for the electron, it is anisotropic for the heavy hole and therefore also for the DE, which is formed by an electronheavy-hole pair. The depolarization times that we measure in the zero magnetic field show that the electron depolarizes much faster than the heavy hole. This observation is explained by the difference between the strong isotropic electron-nucleus hyperfine contact interaction $\left(\gamma_{e}\right)$ and the anisotropic hole-nucleus dipolar hyperfine interactions $\left(\gamma_{h_{Z}}, \gamma_{h_{p}}\right)$. The heavy-hole spin depolarizes faster than the dark-exciton spin due to the electron-hole exchange interaction, which protects the dark-exciton spin from depolarizing. The depolarization of the dark exciton results from residual dark-exciton-bright-exciton mixing. We believe that this mixing can be significantly reduced by increasing the QD symmetry and by avoiding alloying. In this case the dark exciton may form an almost nondephasing electronic spin qubit in a semiconductor environment.

\section{ACKNOWLEDGMENTS}

The support of the Israeli Science Foundation (ISF), and that of the European Research Council (ERC) under the European Union's Horizon 2020 research and innovation programme (Grant Agreement No. 695188) are gratefully acknowledged.

\section{APPENDIX A: HYPERFINE INTERACTION OF THE ELECTRON AND THE HEAVY HOLE}

We outline here a model for describing the temporal evolution of the QD confined central spin polarization in the absence of externally applied magnetic field but in the presence of effective magnetic field generated by the nuclear spins, which comprise the QD. As the central spin 
we consider either the electron or the heavy hole. We then apply the same model also to a central spin formed by the $\mathrm{DE}$ - a long-lived electron-heavy-hole pair, as will be discussed in Appendix B.

As all three cases involve a two-level system (a qubit) they may be described using the Pauli matrices $\sigma_{x}, \sigma_{y}, \sigma_{z}$ and the effective Hamiltonian must take the form

$$
H=\frac{1}{2} \vec{C} \cdot \vec{\sigma}
$$

for some $\vec{C}=\left(C_{x}, C_{y}, C_{z}\right)$. The exact expression of $\vec{C}$ will be different, of course, for each type of central spin.

The hyperfine Fermi-contact interaction between an electron and all the nuclei in the QD is given by [15]

$$
H=\frac{\nu_{0}}{2} \sum\left|\psi_{\mathrm{env}}\left(\vec{r}_{i}\right)\right|^{2} A_{e}^{i} \vec{I}_{i} \cdot \vec{\sigma} .
$$

Here, $\nu_{0}$ is the volume of the unit cell, $\vec{r}_{i}$ and $\vec{I}_{i}$ are the $i$ th nucleus position and its spin operator, $\psi_{\text {env }}(\vec{r})$ describes the electron envelope wave function, and $A_{e}^{i}$ is an effective hyperfine interaction constant between the electron and the specific nucleus in the $\vec{r}_{i}$ position where the index $i$ runs over all the nuclei in the QD. Since $A_{e}^{i}$ depends on the atomic nuclear spin it is much larger for indium atoms than for all other atoms in the QD. Thus, in principle, one can neglect other nuclei contributions. We proceed by defining an expression for the effective magnetic field, which the nuclei apply on the electron. The field, known also as the Overhauser field, is defined as

$$
\vec{B}_{N}=\frac{1}{g_{e} \mu_{B}} \vec{C}_{e}=\frac{\nu_{0}}{g_{e} \mu_{B}} \sum A_{e}^{i}\left|\psi_{\mathrm{env}}\left(\vec{r}_{i}\right)\right|^{2}\left\langle\vec{I}_{i}\right\rangle_{N},
$$

where $g_{e}$ and $\mu_{B}$ are the electron $g$ factor and Bohr magneton, respectively, and $\langle\cdots\rangle_{N}$ denotes a quantum mechanical average over the nuclear spins which interact with the electron.

Assuming that different nuclear spins are not correlated allows one to treat $\vec{B}_{N}(t)$ as having an isotropic Gaussian random distribution satisfying

$$
\langle\vec{B}\rangle=0, \quad\left\langle B_{N x}^{2}\right\rangle=\left\langle B_{N y}^{2}\right\rangle=\left\langle B_{N z}^{2}\right\rangle=\sigma^{2},
$$

where the width of the distribution $\sigma$ is given by [15]

$$
3 \sigma^{2}=\sum \frac{\left(A_{e}^{i}\right)^{2}}{\mu_{B}^{2} g_{e}^{2}} \nu_{0}^{2}\left|\psi_{\mathrm{env}}\left(\vec{r}_{i}\right)\right|^{4} I_{i}\left(I_{i}+1\right) .
$$

It is then convenient to define a modified unitless magnetic field $\overrightarrow{\tilde{B}}=(1 / \sigma) \vec{B}_{N}$. In the following, we simply mark this modified Overhauser field as $\vec{B}$. The electron spin
Hamiltonian can then be expressed by $H=\frac{1}{2} \vec{C}_{e} \cdot \vec{\sigma}$ with $\vec{C}_{e}=\gamma_{e} \vec{B}$, where $\gamma_{e}=g_{e} \mu_{B} \sigma$ is the electron coupling constant in energy units, which we use as a fitting parameter.

While for the electron, $s$-wave molecular symmetry results in a scalar effective coupling $A_{e}^{i}$, for the heavy hole it is described by an anisotropic tensor

$$
\hat{A}_{h}^{i}=\left(\begin{array}{ccc}
A_{h, p}^{i} & & \\
& A_{h, p}^{i} & \\
& & A_{h, z}^{i}
\end{array}\right) .
$$

Where the in plane dipole-dipole interaction constant $A_{h, p}^{i}$ does not strictly vanish for the heavy hole due to mixing with the light hole [41]. Therefore, for the heavy hole we define $C_{z}=\gamma_{h_{z}} B_{z}, C_{x, y}=\gamma_{h_{p}} B_{x, y}$, where $\gamma_{h_{z}}>\gamma_{h_{p}}$ are also fitting parameters. Strictly speaking, the field $\vec{B}$ appearing here is not exactly the same one as in the electron case. This is due to differences in relative weighting of various nuclei between electron and hole wave functions. For our purpose, however, it is sufficient that the fields have the same Gaussian statistics. For the moment we allow the functional relation between $\vec{C}$ and $\vec{B}$ to be arbitrary and since our discussion is independent of these relations, it applies to all three cases.

At short times $\vec{B}$ and hence also $\vec{C}$ can be treated as time independent and one readily find the solution

$$
\begin{aligned}
\vec{S}(t)= & \frac{\vec{S}_{0} \cdot \vec{C}}{C^{2}} \vec{C}+\left(\vec{S}_{0}-\frac{\vec{S}_{0} \cdot \vec{C}}{C^{2}} \vec{C}\right) \cos \left(\frac{C}{\hbar} t\right) \\
& -\frac{\vec{S}_{0} \times \vec{C}}{C} \sin \left(\frac{C}{\hbar} t\right),
\end{aligned}
$$

where $\vec{S}_{0}=\vec{S}(0)$ is the central spin initial value. The first term is time independent and survives for long times. Upon averaging over the random ensemble of possible $\vec{C}$ s one typically finds that the oscillating terms turn into exponentially decaying transients, relevant at short times only. In practice, the last term usually vanishes by symmetry under $\vec{C} \rightarrow-\vec{C}$. In particular it applies to our experiments, which are carried out in the absence of externally applied magnetic field. Therefore, in the following we disregard this term.

At longer times, we use the adiabatic approximation and assume that the central spin follows the direction of $\vec{C}$, while the rapidly rotating components orthogonal to $\vec{C}$ average to zero. We can therefore write

$$
\vec{S}(t)=\left[\vec{S}_{0} \cdot \hat{C}(0)\right] \hat{C}(t)=\frac{\vec{S}_{0} \cdot \vec{C}(0)}{C(0) C(t)} \vec{C}(t) .
$$


For small $t$ this clearly coincides with the first term of Eq. (A1). As the other terms of Eq. (A1) vanish at long times one sees that the two relations, Eqs. (A1) and (A2), can be combined into an expression which applies at arbitrary time $t$ :

$$
\begin{aligned}
\vec{S}(t)= & \frac{\vec{S}_{0} \cdot \vec{C}(0)}{C(0) C(t)} \vec{C}(t) \\
& +\left(\vec{S}_{0}-\frac{\vec{S}_{0} \cdot \vec{C}(0)}{C(0)^{2}} \vec{C}(0)\right) \cos \left(\frac{C(0)}{\hbar} t\right) .
\end{aligned}
$$

The Gaussian probability density corresponding to the dimensionless Overhauser field at a given moment is given by

$$
d P_{1}=\frac{1}{(2 \pi)^{3 / 2}} \exp \left(-\frac{1}{2} B^{2}\right) d^{3} B
$$

Assuming further that

$$
\left\langle B_{i}\left(t_{1}\right) B_{j}\left(t_{2}\right)\right\rangle=\delta_{i j} f\left(t_{2}-t_{1}\right)
$$

[consistency requires $f(0)=1$ ], we can write the joint probability density of $\vec{B}_{1}=\vec{B}(0)$ and $\vec{B}_{2}=\vec{B}(t)$ as

$$
\begin{aligned}
d P_{2}= & \frac{d^{3} B_{1} d^{3} B_{2}}{\left(2 \pi \sqrt{1-f(t)^{2}}\right)^{3}} \\
& \times \exp \left(-\frac{1}{2}\left[B_{1}^{2}+B_{2}^{2}-2 f(t) \vec{B}_{1} \cdot \vec{B}_{2}\right] /\left[1-f(t)^{2}\right]\right) .
\end{aligned}
$$

Using the probability distributions, Eqs. (A4) and (A5), we can write the average central spin evolution as

$$
\begin{aligned}
\langle\vec{S}(t)\rangle= & \int \frac{\vec{S}_{0} \cdot \vec{C}(0)}{C(0) C(t)} \vec{C}(t) d P_{2} \\
& +\int\left(\vec{S}_{0}-\frac{\vec{S}_{0} \cdot \vec{C}}{C^{2}} \vec{C}\right) \cos \left(\frac{C}{\hbar} t\right) d P_{1} .
\end{aligned}
$$

Actual computation of the integrals requires using the specific functional relation between $\vec{B}$ and $\vec{C}$.

For the electron as the central spin, we simply substitute $\vec{C}=\gamma_{e} \vec{B}$ and $\vec{S}_{0}=\hat{z}$ in Eq. (A6) and obtain integrals which can be evaluated analytically $[15,33]$, resulting in

$$
\begin{aligned}
\left\langle S_{z}\right\rangle= & \frac{2}{3}\left[1-\left(\frac{\gamma_{e} t}{\hbar}\right)^{2}\right] e^{-(1 / 2)\left(\gamma_{e} t / \hbar\right)^{2}} \\
& +\frac{2}{3 \pi}\left[\sqrt{\frac{1}{f(t)^{2}}-1}+\left(2-\frac{1}{f(t)^{2}}\right) \arcsin [f(t)]\right] .
\end{aligned}
$$

For the heavy hole as the central spin we have $C_{z}=\alpha B_{z}$, $C_{x, y}=\beta B_{x, y}$ with $\alpha=\gamma_{h_{z}} \beta=\gamma_{h_{p}}$ In this case $\left\langle S_{z}\right\rangle$ is given according to Eq. (A6) by a sum of two rather complicated integrals. The second term of Eq. (A6) can be reduced into a one-dimensional (1D) integral which we then calculate numerically

$\frac{\beta^{2}}{\left(\alpha^{2}-\beta^{2}\right)^{3 / 2}} \int_{\beta}^{\alpha} d \xi \frac{\left(\alpha^{2}-\xi^{2}\right)\left(1-\sigma^{2} \xi^{2} t^{2}\right)}{\xi \sqrt{\xi^{2}-\beta^{2}}} e^{-(1 / 2) \sigma^{2} \xi^{2} t^{2}}$.

The first term of Eq. (A6) is a more complicated 6D integral. If we use the following shortcuts:

$$
\begin{aligned}
& a_{0}=\sqrt{\left(\frac{\cos ^{2} \theta}{\alpha^{2}}+\frac{\sin ^{2} \theta}{\beta^{2}}\right)\left(\frac{\cos ^{2} \theta^{\prime}}{\alpha^{2}}+\frac{\sin ^{2} \theta^{\prime}}{\beta^{2}}\right),} \\
& a_{1}=f(t)\left(\frac{\cos \theta \cos \theta^{\prime}}{\alpha^{2}}+\frac{\sin \theta \sin \theta^{\prime}}{\beta^{2}} \cos \varphi\right), \\
& A_{0}=\frac{\left[1-f(t)^{2}\right]^{3 / 2}}{4 \pi^{2} \alpha^{2} \beta^{4}} \sin (2 \theta) \sin \left(2 \theta^{\prime}\right),
\end{aligned}
$$

then the $6 \mathrm{D}$ integral can be reduced into a $3 \mathrm{D}$ one

$$
\begin{gathered}
\int_{0}^{2 \pi} d \varphi \int_{0}^{\pi / 2} d \theta \int_{0}^{\pi / 2} d \theta^{\prime} A_{0}\left[\frac{3 a_{1}}{\left(a_{0}^{2}-a_{1}^{2}\right)^{2}}\right. \\
\left.+\frac{a_{0}^{2}+2 a_{1}^{2}}{\left(a_{0}^{2}-a_{1}^{2}\right)^{5 / 2}} \arcsin \left(a_{1} / a_{0}\right)\right],
\end{gathered}
$$

which we then calculate numerically. The function $f(t)$ is essentially the Overhauser field-time correlator. An appropriate model for the evolution of the Overhauser field is required for its evaluation.

By using $\vec{B}=\left(\nu_{0} / g_{e} \mu_{B} \sigma\right) \sum A_{i}\left|\psi_{\text {env }}\left(\vec{r}_{i}\right)\right|^{2} \vec{I}_{i}$ one obtains

$$
\begin{aligned}
3 \sigma^{2} f(t) & =\langle\vec{B}(0) \cdot \vec{B}(t)\rangle \\
& =\sum\left(\frac{\nu_{0}}{g_{e} \mu_{B} \sigma} A_{i}^{2}\left|\psi_{\mathrm{env}}\left(\vec{r}_{i}\right)\right|^{2}\right)^{2}\left\langle\vec{I}_{i}(0) \cdot \vec{I}_{i}(t)\right\rangle .
\end{aligned}
$$

A particularly simple model assumes that the Overhauser field evolution is dominated by the quadrupole interaction of the nuclear spins $[31,33]$. Though more complicated models exist as well $[15,33,58]$, this model permits analytical solutions.

Within this model each nuclear spin $\vec{I}_{k}=\vec{I}$ evolves independently of the others by a Hamiltonian of the form $H_{Q}=V_{i j} I_{i} I_{j}$ with random $V_{i j}=V_{j i}$ which relates to the local electric field gradients (EFG) [31]. We take the initial state of the nuclear spin to be random and we average over the corresponding wave function, thereby obtaining

$$
\langle\vec{I}(0) \cdot \vec{I}(t)\rangle \propto \operatorname{Tr}\left(\vec{I} \cdot e^{i H_{Q} t} \vec{I} e^{-i H_{Q^{t}}}\right) .
$$


As different nuclear spins have different EFG we obtain the Overhauser correlator $f(t)$ by averaging over the $V_{i j}$ terms. We take (as common in random matrix theory) the elements of the symmetric matrix $V_{i j}$ to be independent Gaussian random variables of variance $\gamma_{Q}^{2}$. Up to overall normalization we obtain

$$
f(t) \propto \int d V e^{-\operatorname{Tr} V^{2} /\left(2 \gamma_{Q}^{2}\right)} \operatorname{Tr}\left(\vec{I} \cdot e^{i H_{Q} t} \vec{I} e^{-i H_{Q} t}\right) .
$$

Noting that $V$ can be taken as a traceless tensor and in addition using its polar decomposition, we reduce the above expression into a two-dimensional integral which we express as

$$
\begin{aligned}
f(t) \propto & \int d x_{1} d x_{2} d x_{3} \delta\left(\sum x_{i}\right) \prod_{i<j}\left|x_{i}-x_{j}\right| e^{\left[-\left(x_{1}^{2}+x_{2}^{2}+X_{3}^{2}\right)\right] / 2 \gamma_{Q}^{2}} \\
& \cdot \operatorname{Tr}\left(\vec{I} \cdot e^{i \sum x_{i} I_{i}^{2} t} \vec{I} e^{-i \sum x_{i} I_{i}^{2} t}\right) .
\end{aligned}
$$

For $I=(3 / 2)$ we evaluate this expression and obtain

$$
\begin{aligned}
& f_{3 / 2}(t) \propto \int_{0}^{\infty} d x x^{4} e^{-x^{2} /\left(2 \gamma_{Q}^{2}\right)}[3+2 \cos (\sqrt{6} x t)], \\
& f_{3 / 2}(t)=\frac{3}{5}+\frac{2}{5}\left[1-2\left(\frac{\gamma_{Q} t}{\hbar}\right)^{2}+12\left(\frac{\gamma_{Q} t}{\hbar}\right)^{4}\right] e^{-3\left(\gamma_{Q} t / \hbar\right)^{2}} .
\end{aligned}
$$

For higher values of the nuclear spin $I$, we calculate $f_{I}(t)$ numerically as a function of the dimensionless product $\gamma_{Q} t / \hbar$. This gives a qualitatively similar result to Eq. (A9) with some modifications. Since our QD contains $I=3 / 2$, $I=9 / 2$, and $I=1 / 2$ we averaged over these values using the relative nuclear abundance multiplied by the squared nuclear moments as weights. In Fig. 5 we display the normalized Overhauser correlator for various types of nuclear spins in the QD. For simplicity we assume the same $\gamma_{Q}$ for all atom types. In practice the indium contribution dominates the average due to its large magnetic moment. This may explain why the quadrupolar coupling that we deduce for the InAsP QD that we studied is larger by about a factor of 3 than that reported by Bechtold et al. [33] for InGaAs QDs. In both QDs the nuclear field is mostly due to the indium atoms which have a very large nuclear moment $[I=(9 / 2)]$. The quadrupolar coupling is then due to the electric field gradient (EFG) generated by neighboring atoms of the indium. In InGaAs QDs all four nearest neighbors of the indium atom are identical arsenide atoms and therefore their contribution to the EFG is canceled by symmetry. In InAsP QDs, in contrast, the nearest neighbors of an indium atom can be either arsenide or phosphide atoms, and hence they may generate a nonzero net EFG. The InAsP EFG is therefore larger than that in InGaAs QDs where the main

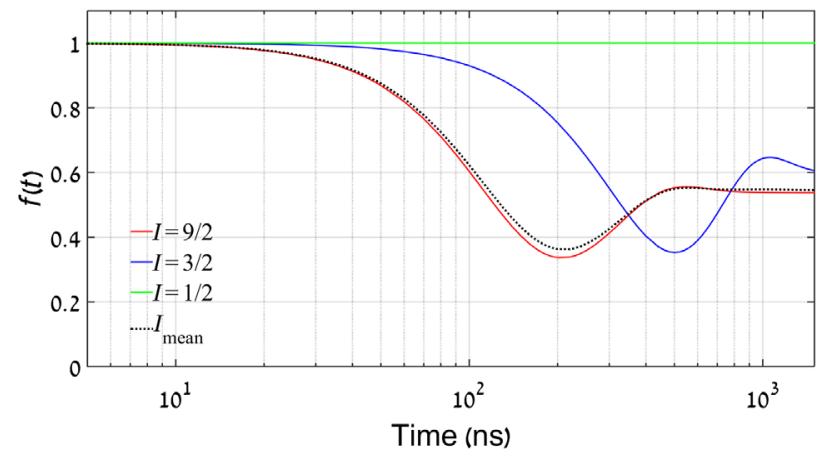

FIG. 5. The calculated normalized Overhauser correlator $f_{I}(t)$ for the various types of nuclear spins which comprise the QD. $I_{\text {mean }}$ is the mean value of the correlator taking into account the isotopic abundances weighted by their squared magnetic moments.

contribution to the EFG comes from next nearest neighbors atoms only (either indium or gallium atoms). In addition, the indium content in InAsP QDs is at least a factor of 2 larger than the Ga-enriched InGaAs QDs studied in Ref. [33], as judged by their emission wavelength.

\section{APPENDIX B: HYPERFINE INTERACTION OF THE DARK EXCITON}

The spin projection $\left[S_{z}(t)\right]$ of the DE strongly depends on the electron-hole exchange interaction.

We describe the DE qubit by its two spin states: $|\Uparrow \uparrow\rangle$ and $|\Downarrow \downarrow\rangle$, with $J_{z}=+2$, and -2 , respectively. While the $\mathrm{DE}$ interaction with the $z$ component $B_{z}$ of the Overhauser field is similar to that of a spin $1 / 2$ central spin (up to multiplicative constant [30]), its interaction with the $B_{x, y}$ components is very different. Strictly speaking, a standard $\vec{B} \cdot \vec{J}$ Hamiltonian would have to act four times in order to flip a $J_{z}=2$ state into a $J_{Z}=-2$ state. However, if one fully considers the electron-hole exchange interaction, this is not the case. In the bright and dark excitons basis $\{|\Uparrow \downarrow\rangle,|\Downarrow \uparrow\rangle,|\Uparrow \uparrow\rangle,|\Downarrow \downarrow\rangle\}$, the exchange interaction can be expressed as [30,59]

$$
\frac{1}{2}\left(\begin{array}{cccc}
\Delta_{0} & \Delta_{1}^{*} & \Delta_{3} & \Delta_{4} \\
\Delta_{1} & \Delta_{0} & -\Delta_{4}^{*} & -\Delta_{3}^{*} \\
\Delta_{3}^{*} & -\Delta_{4} & -\Delta_{0} & \Delta_{2}^{*} \\
\Delta_{4}^{*} & -\Delta_{3} & \Delta_{2} & -\Delta_{0}
\end{array}\right),
$$

where $\Delta_{0}$ is the isotropic exchange interaction. It is a real number, which defines the energy splitting between the DE and BE eigenstates. It is measured to be $\Delta_{0}=260 \mu \mathrm{eV}$ for the QD under study. The term

$$
\Delta_{1}=\delta_{1} \exp \left(i 2 \theta_{1}\right)
$$


is the anisotropic long-range exchange interaction. Here, $\delta_{1}$ is a positive number defining the magnitude of the bright exciton (BE) fine-structure splitting (FSS) [21], and $\theta_{1}$ defines the directions of the two cross-linearly-polarized components of the $\mathrm{BE}$ spectral lines with respect to the crystallographic directions [30].

$$
\Delta_{2}=\delta_{2} \exp \left(i 2 \theta_{2}\right)
$$

describes the FSS of the dark exciton. Here, $\delta_{2}$ and $\theta_{2}$ are real numbers mainly given by the short-range anisotropic exchange interaction.

$$
\Delta_{3}=\delta_{3} \exp \left(i 2 \theta_{3}\right)
$$

and

$$
\Delta_{4}=\delta_{4} \exp \left(i 2 \theta_{4}\right)
$$

are also long-range exchange interactions that couple between the DE and BE states.

Strictly speaking, for a $C_{3 v}$ symmetrical $\mathrm{QD}, \delta_{1}, \delta_{2}, \delta_{3}$, and $\delta_{4}$ are all expected to vanish [60]. To within our experimental uncertainty, we find it to be true only for $\delta_{2}(<0.1 \mu \mathrm{eV})$, since it results from the short-range exchange interaction and therefore affected mainly by the symmetry of the QD's unit cells [30]. Structural deviations of the QD from symmetry such as composition fluctuations, or faceting, destroy the QD long-range symmetry, without affecting its unit cell symmetry. Therefore, they will result in finite $\delta_{1}, \delta_{3}$, and $\delta_{4}$. Indeed, we measure $\delta_{1}=18 \mu \mathrm{eV}$ by polarization-sensitive spectroscopy, and estimate $\delta_{3} \backsim \delta_{4} \backsim 15 \mu \mathrm{eV}$ by measuring the DE radiative lifetime, and verifying the fact that the DE weak absorption line is linearly polarized in plane [29].

Since $\left|\Delta_{3}\right|=\left|\Delta_{4}\right| \neq 0$, these terms induce coupling between the DE and BE states. We define $\left(\Delta_{3}+\Delta_{4}\right) / 2$ $\triangleq \Delta_{\mathrm{DB}}$, and since $\left|\Delta_{\mathrm{DB}}\right| \ll \Delta_{0}$, the modified DE eigenstates remain almost degenerate such that the symmetric and antisymmetric spin combinations are expressed as

$$
\begin{aligned}
\left|\mathrm{DE}_{A S}\right\rangle & =N_{A S}\left[\frac{|\Uparrow \uparrow\rangle-|\Downarrow \downarrow\rangle}{\sqrt{2}}-\frac{\Delta_{\mathrm{DB}}}{\Delta_{0}} \frac{|\Uparrow \downarrow\rangle+|\Downarrow \uparrow\rangle}{\sqrt{2}}\right], \\
\left|\mathrm{DE}_{S}\right\rangle & =\frac{|\Uparrow \uparrow\rangle+|\Downarrow \downarrow\rangle}{\sqrt{2}},
\end{aligned}
$$

where $N_{A S} \sim 1$ is a normalization constant. This also agrees with the experimental observation that the DE has only one weak optically active eigenstate, which is linearly polarized like the symmetric BE eigenstate $[59,61,62]$. The mixing term is sufficient to provide a nuclear-field-dependent flipping of either the heavy hole or the electron in order to change the DE state from the $|\Uparrow \uparrow\rangle$ to the $|\Downarrow \downarrow\rangle$, or vice versa. Hence, the interaction is linear in the nuclear magnetic field and the DE Hamiltonian takes the form $H=(1 / 2) \vec{C}^{(\mathrm{DE})} \cdot \vec{\sigma}$ with

$$
\begin{aligned}
& C_{x, y}^{(\mathrm{DE})}=\frac{2 \operatorname{Im}\left[\Delta_{\mathrm{DB}}\right]}{\Delta_{0}}\left(C_{x, y}^{(e)}+C_{x, y}^{(h)}\right), \\
& C_{z}^{(\mathrm{DE})}=\left(C_{z}^{(e)}+C_{z}^{(h)}\right) .
\end{aligned}
$$

If we express $\vec{C}_{e}, \vec{C}_{h}$ as earlier in terms of the same dimensionless $\vec{B}$, we conclude

$$
\begin{aligned}
& \gamma_{\mathrm{D} E_{p}}=\frac{2 \operatorname{Im}\left[\Delta_{\mathrm{DB}}\right]}{\Delta_{0}}\left(\gamma_{e}+\gamma_{h_{p}}\right), \\
& \gamma_{\mathrm{DE}_{z}}=\gamma_{e}+\gamma_{h_{z}}=\gamma_{e}-\left|\gamma_{h_{z}}\right|,
\end{aligned}
$$

where we used the fact that $\gamma_{h_{z}}<0$ [63].

$\operatorname{Im}\left[\Delta_{\mathrm{DB}}\right] \leqq \delta_{3} \approx 15 \mu \mathrm{eV}$ provides an estimate for $\gamma_{\mathrm{D} E_{p}}$ (see Table. I), and we note here that the fields $\vec{C}_{e}$ and $\vec{C}_{h}$ experienced by the electron and by the heavy hole, respectively, may not be in perfect correlation [17]. This is expected to reduce their interference effects, making $\gamma_{\mathrm{DE}_{z}}$ slightly larger and $\gamma_{\mathrm{D} E_{p}}$ slightly smaller than the above estimations.

The DE Hamiltonian as explained above is linear in $B$ and anisotropic, much like the one for the heavy-hole spin. Consequently $\left\langle S_{z}(t)\right\rangle$ is derived in a similar way to that of the heavy-hole spin in Eqs. (A7) and (A8) by replacing $\alpha=\gamma_{\mathrm{DE}_{z}}$ and $\beta=\gamma_{\mathrm{D} E_{p}}$.

[1] D. Loss and D. P. DiVincenzo, Quantum Computation with Quantum Dots, Phys. Rev. A 57, 120 (1998).

[2] H. J. Kimble, The Quantum Internet, Nature (London) 453, 1023 (2008).

[3] J. Berezovsky, M. H. Mikkelsen, N. G. Stoltz, L. A. Coldren, and D.D. Awschalom, Picosecond Coherent Optical Manipulation of a Single Electron Spin in a Quantum Dot, Science 320, 349 (2008).

[4] D. Press, T. D. Ladd, B. Zhang, and Y. Yamamoto, Complete Quantum Control of a Single Quantum Dot Spin Using Ultrafast Optical Pulses, Nature (London) 456, 218 (2008).

[5] A. J. Ramsay, S. J. Boyle, R. S. Kolodka, J. B. B. Oliveira, J. Skiba-Szymanska, H. Y. Liu, M. Hopkinson, A. M. Fox, and M. S. Skolnick, Fast Optical Preparation, Control, and Readout of a Single Quantum Dot Spin, Phys. Rev. Lett. 100, 197401 (2008).

[6] Quantum Dots for Quantum Information Technologies, edited by P. Michler (Springer International Publishing, Basel, 2017).

[7] A. Imamoglu, D. D. Awschalom, G. Burkard, D. P. DiVincenzo, D. Loss, M. Sherwin, and A. Small, Quantum Information Processing Using Quantum Dot Spins and Cavity QED, Phys. Rev. Lett. 83, 4204 (1999). 
[8] W. B. Gao, P. Fallahi, E. Togan, J. Miguel-Sanchez, and A. Imamoglu, Observation of Entanglement between a Quantum Dot Spin and a Single Photon, Nature (London) 491, 426 (2012).

[9] K. De Greve, L. Yu, P. L. McMahon, J. S. Pelc, C. M. Natarajan, N. Y. Kim, E. Abe, S. Maier, C. Schneider, M. Kamp, S. Höfling, R. H. Hadfield, A. Forchel, M. M. Fejer, and Y. Yamamoto, Quantum-Dot Spin-Photon Entanglement via Frequency Downconversion to Telecom Wavelength, Nature (London) 491, 421 (2012).

[10] J. R. Schaibley, A. P. Burgers, G. A. McCracken, L.-M. Duan, P. R. Berman, D. G. Steel, A. S. Bracker, D. Gammon, and L.J. Sham, Demonstration of Quantum Entanglement between a Single Electron Spin Confined to an InAs Quantum Dot and a Photon, Phys. Rev. Lett. 110, 167401 (2013).

[11] I. Schwartz, D. Cogan, E. R. Schmidgall, Y. Don, L. Gantz, O. Kenneth, N. H. Lindner, and D. Gershoni, Deterministic Generation of a Cluster State of Entangled Photons, Science 354, 434 (2016).

[12] N. Bloembergen, E. M. Purcell, and R. V. Pound, Relaxation Effects in Nuclear Magnetic Resonance Absorption, Phys. Rev. 73, 679 (1948).

[13] P. A. Abragam and A. Abragam, The Principles of Nuclear Magnetism, Comparative Pathobiology-Studies in the PostModern Theory of Education (Clarendon Press, Oxford, 1961).

[14] D. Gammon, A. L. Efros, T. A. Kennedy, M. Rosen, D. S. Katzer, D. Park, S. W. Brown, V. L. Korenev, and I. A. Merkulov, Electron and Nuclear Spin Interactions in the Optical Spectra of Single GaAs Quantum Dots, Phys. Rev. Lett. 86, 5176 (2001).

[15] I. A. Merkulov, A. L. Efros, and M. Rosen, Electron Spin Relaxation by Nuclei in Semiconductor Quantum Dots, Phys. Rev. B 65, 205309 (2002).

[16] A. V. Khaetskii, D. Loss, and L. Glazman, Electron Spin Decoherence in Quantum Dots due to Interaction with Nuclei, Phys. Rev. Lett. 88, 186802 (2002).

[17] J. Fischer, W. A. Coish, D. V. Bulaev, and D. Loss, Spin Decoherence of a Heavy Hole Coupled to Nuclear Spins in a Quantum Dot, Phys. Rev. B 78, 155329 (2008).

[18] J. Y. Marzin, J. M. Gérard, A. Izraël, D. Barrier, and G. Bastard, Photoluminescence of Single InAs Quantum Dots Obtained by Self-Organized Growth on GaAs, Phys. Rev. Lett. 73, 716 (1994).

[19] E. Dekel, D. Gershoni, E. Ehrenfreund, D. Spektor, J. M. Garcia, and P. M. Petroff, Multiexciton Spectroscopy of a Single Self-Assembled Quantum Dot, Phys. Rev. Lett. 80, 4991 (1998).

[20] D. P. DiVincenzo, The Physical Implementation of Quantum Computation, Fortschr. Phys. 48, 771 (2000).

[21] E. L. Ivchenko, Optical Spectroscopy of Semiconductor Nanostructures (Alpha Science, Oxford, 2005).

[22] D. Brunner, B. D. Gerardot, P. A. Dalgarno, G. Wüst, K. Karrai, N. G. Stoltz, P. M. Petroff, and R. J. Warburton, A Coherent Single-Hole Spin in a Semiconductor, Science 325, 70 (2009).

[23] K.n De Greve, P. L. McMahon, D. Press, T. D. Ladd, D. Bisping, C. Schneider, M. Kamp, L. Worschech, S. Höfling, A. Forchel, and Y. Yamamoto, Ultrafast Coherent Control and Suppressed Nuclear Feedback of a Single Quantum Dot Hole Qubit, Nat. Phys. 7, 872 (2011).

[24] Y. Benny, S. Khatsevich, Y. Kodriano, E. Poem, R. Presman, D. Galushko, P. M. Petroff, and D. Gershoni, Coherent Optical Writing and Reading of the Exciton Spin State in Single Quantum Dots, Phys. Rev. Lett. 106, 040504 (2011).

[25] Y. Kodriano, I. Schwartz, E. Poem, Y. Benny, R. Presman, T. A. Truong, P. M. Petroff, and D. Gershoni, Complete Control of a Matter Qubit Using a Single Picosecond Laser Pulse, Phys. Rev. B 85, 241304 (2012).

[26] E. Poem, O. Kenneth, Y. Kodriano, Y. Benny, S. Khatsevich, J. E. Avron, and D. Gershoni, Optically Induced Rotation of an Exciton Spin in a Semiconductor Quantum Dot, Phys. Rev. Lett. 107, 087401 (2011).

[27] J. McFarlane, P. A. Dalgarno, B. D. Gerardot, R. H. Hadfield, R. J. Warburton, K. Karrai, A. Badolato, and P. M. Petroff, Gigahertz. Bandwidth Electrical Control over a Dark Exciton-Based Memory Bit in a Single Quantum Dot, Appl. Phys. Lett. 94, 093113 (2009).

[28] E. Poem, Y. Kodriano, C. Tradonsky, N. H. Lindner, B. D. Gerardot, P. M. Petroff, and D. Gershoni, Accessing the Dark Exciton with Light, Nat. Phys. 6, 993 (2010).

[29] I. Schwartz, E. R. Schmidgall, L. Gantz, D. Cogan, E. Bordo, Y. Don, M. Zielinski, and D. Gershoni, Deterministic Writing and Control of the Dark Exciton Spin Using Single Short Optical Pulses, Phys. Rev. X 5, 011009 (2015).

[30] M. Bayer, G. Ortner, O. Stern, A. Kuther, A. A. Gorbunov, A. Forchel, P. Hawrylak, S. Fafard, K. Hinzer, T. L. Reinecke, S. N. Walck, J. P. Reithmaier, F. Klopf, and F. Schäfer, Fine Structure of Neutral and Charged Excitons in Self-Assembled InGaAs/AlGaAs Quantum Dots, Phys. Rev. B 65, 195315 (2002).

[31] N. A. Sinitsyn, Y. Li, S. A. Crooker, A. Saxena, and D. L. Smith, Role of Nuclear Quadrupole Coupling on Decoherence and Relaxation of Central Spins in Quantum Dots, Phys. Rev. Lett. 109, 166605 (2012).

[32] R. V. Pound, Nuclear Electric Quadrupole Interactions in Crystals, Phys. Rev. 79, 685 (1950).

[33] A. Bechtold, D. Rauch, F. Li, T. Simmet, P.-L. Ardelt, A. Regler, K. Muller, N. A. Sinitsyn, and J. J. Finley, ThreeStage Decoherence Dynamics of an Electron Spin Qubit in an Optically Active Quantum Dot, Nat. Phys. 11, 1005 (2015).

[34] R. Stockill, C. Le Gall, C. Matthiesen, L. Huthmacher, E. Clarke, M. Hugues, and M. Atatüre, Quantum Dot Spin Coherence Governed by a Strained Nuclear Environment, Nat. Commun. 7, 12745 (2016).

[35] S. I. Erlingsson and Y. V. Nazarov, Evolution of Localized Electron Spin in a Nuclear Spin Environment, Phys. Rev. B 70, 205327 (2004).

[36] P.-F. Braun, X. Marie, L. Lombez, B. Urbaszek, T. Amand, P. Renucci, V. K. Kalevich, K. V. Kavokin, O. Krebs, P. Voisin, and Y. Masumoto, Direct Observation of the Electron Spin Relaxation Induced by Nuclei in Quantum Dots, Phys. Rev. Lett. 94, 116601 (2005).

[37] A. Bracker, E. Stinaff, D. Gammon, M. Ware, J. Tischler, A. Shabaev, A. Efros, D. Park, D. Gershoni, V. Korenev, and I. Merkulov, Optical Pumping of the Electronic and Nuclear Spin of Single Charge-Tunable Quantum Dots, Phys. Rev. Lett. 94, 047402 (2005). 
[38] H. Bluhm, S. Foletti, I. Neder, M. Rudner, D. Mahalu, V. Umansky, and A. Yacoby, Dephasing Time of GaAs Electron-Spin Qubits Coupled to a Nuclear Bath Exceeding 200 Microseconds, Nat. Phys. 7, 109 (2011).

[39] C.-Y. Lu, Y. Zhao, A. N. Vamivakas, C. Matthiesen, S. Fält, A. Badolato, and M. Atatüre, Direct Measurement of Spin Dynamics in InAs/GaAs Quantum Dots Using TimeResolved Resonance Fluorescence, Phys. Rev. B 81, 035332 (2010).

[40] B. D. Gerardot, D. Brunner, P. A. Dalgarno, P. Öhberg, S. Seidl, M. Kroner, K. Karrai, N. G. Stoltz, P. M. Petroff, and R. J. Warburton, Optical Pumping of a Single Hole Spin in a Quantum Dot, Nature (London) 451, 441 (2008).

[41] B. Eble, C. Testelin, P. Desfonds, F. Bernardot, A. Balocchi, T. Amand, A. Miard, A. Lemaître, X. Marie, and M. Chamarro, Hole-Nuclear Spin Interaction in Quantum Dots, Phys. Rev. Lett. 102, 146601 (2009).

[42] F. Fras, B. Eble, P. Desfonds, F. Bernardot, C. Testelin, M. Chamarro, A. Miard, and A. Lemaître, Hole-Spin Initialization and Relaxation Times in InAs/GaAs Quantum Dots, Phys. Rev. B 84, 125431 (2011).

[43] Y. Li, N. Sinitsyn, D. L. Smith, D. Reuter, A. D. Wieck, D. R. Yakovlev, M. Bayer, and S. A. Crooker, Intrinsic Spin Fluctuations Reveal the Dynamical Response Function of Holes Coupled to Nuclear Spin Baths in (In, Ga)As Quantum Dots, Phys. Rev. Lett. 108, 186603 (2012).

[44] E. A. Chekhovich, M. M. Glazov, A. B. Krysa, M. Hopkinson, P. Senellart, A. Lemaître, M. S. Skolnick, and A. I. Tartakovskii, Element-Sensitive Measurement of the Hole-Nuclear Spin Interaction in Quantum Dots, Nat. Phys. 9, 74 (2013).

[45] L. Huthmacher, R. Stockill, E. Clarke, M. Hugues, C. Le Gall, and M. Atatüre, Coherence of a Dynamically Decoupled Quantum-Dot Hole Spin, Phys. Rev. B 97, 241413 (2018).

[46] W. D. Knight, Nuclear Magnetic Resonance Shift in Metals, Phys. Rev. 76, 1259 (1949).

[47] A. W. Overhauser, Polarization of Nuclei in Metals, Phys. Rev. 92, 411 (1953).

[48] A. Greilich, A. Shabaev, D. R. Yakovlev, Al. L. Efros, I. A. Yugova, D. Reuter, A. D. Wieck, and M. Bayer, NucleiInduced Frequency Focusing of Electron Spin Coherence, Science 317, 1896 (2007).

[49] E. Barnes and S. E. Economou, Electron-Nuclear Dynamics in a Quantum Dot under Nonunitary Electron Control, Phys. Rev. Lett. 107, 047601 (2011).

[50] S. G. Carter, S. E. Economou, A. Greilich, E. Barnes, T. Sweeney, A. S. Bracker, and D. Gammon, Strong Hyperfine-Induced Modulation of an Optically Driven Hole Spin in an InAs Quantum Dot, Phys. Rev. B 89, 075316 (2014).
[51] D. Dalacu, A. Kam, D. G. Austing, X. Wu, J. Lapointe, G. C. Aers, and P. J. Poole, Selective-Area Vapour-LiquidSolid Growth of InP Nanowires, Nanotechnology 20, 395602 (2009).

[52] D. Dalacu, K. Mnaymneh, J. Lapointe, X. Wu, P. J. Poole, G. Bulgarini, V. Zwiller, and M. E. Reimer, Ultraclean Emission from InAsP Quantum Dots in Defect-Free Wurtzite InP Nanowires, Nano Lett. 12, 5919 (2012).

[53] G. Bulgarini, M. E. Reimer, M. B. Bavinck, K. D. Jöns, D. Dalacu, P. J. Poole, E. P. A. M. Bakkers, and V. Zwiller, Nanowire Waveguides Launching Single Photons in a Gaussian Mode for Ideal Fiber Coupling, Nano Lett. 14, 4102 (2014).

[54] Y. Benny, Y. Kodriano, E. Poem, D. Gershoni, T. A. Truong, and P. M. Petroff, Excitation Spectroscopy of Single Quantum Dots at Tunable Positive, Neutral, and Negative Charge States, Phys. Rev. B 86, 085306 (2012).

[55] E. R. Schmidgall, I. Schwartz, D. Cogan, L. Gantz, T. Heindel, S. Reitzenstein, and D. Gershoni, All-Optical Depletion of Dark Excitons from a Semiconductor Quantum Dot, Appl. Phys. Lett. 106, 193101 (2015).

[56] I. Schwartz, D. Cogan, E. R. Schmidgall, L. Gantz, Y. Don, M. Zieliński, and D. Gershoni, Deterministic Coherent Writing of a Long-Lived Semiconductor Spin Qubit Using One Ultrafast Optical Pulse, Phys. Rev. B 92, 201201 (2015).

[57] B. Gotschy, G. Denninger, H. Obloh, W. Wilkening, and J. Schneider, Overhauser Shift and Dynamic Nuclear Polarization in InP, Solid State Commun. 71, 629 (1989).

[58] K. A. Al-Hassanieh, V. V. Dobrovitski, E. Dagotto, and B. N. Harmon, Numerical Modeling of the Central Spin Problem Using the Spin-Coherent-State P Representation, Phys. Rev. Lett. 97, 037204 (2006).

[59] Y. Don, M. Zielinski, and D. Gershoni, The Optical Activity of the Dark Exciton, arXiv:1601.05530v1.

[60] M. A. Dupertuis, K. F. Karlsson, D. Y. Oberli, E. Pelucchi, A. Rudra, P. O. Holtz, and E. Kapon, Symmetries and the Polarized Optical Spectra of Exciton Complexes in Quantum Dots, Phys. Rev. Lett. 107, 127403 (2011).

[61] M. Zieliński, Y. Don, and D. Gershoni, Atomistic Theory of Dark Excitons in Self-Assembled Quantum Dots of Reduced Symmetry, Phys. Rev. B 91, 085403 (2015).

[62] E. R. Schmidgall, I. Schwartz, D. Cogan, L. Gantz, Y. Don, and D. Gershoni, Coherent Control of Dark Excitons in Semiconductor Quantum Dots, in Quantum Dots for Quantum Information Technologies (Springer International Publishing, Basel, 2017), pp. 123-164.

[63] B. J. Witek, R. W. Heeres, U. Perinetti, E. P. A. M. Bakkers, L. P. Kouwenhoven, and V. Zwiller, Measurement of the $g$-Factor Tensor in a Quantum Dot and Disentanglement of Exciton Spins, Phys. Rev. B 84, 195305 (2011). 